\title{
A gain-scheduled LPV control for oxygen stoichiometry regulation in PEM fuel cell systems
}

\author{
Fernando D. Bianchi, Cristian Kunusch, Member, IEEE, Carlos Ocampo-Martinez, Senior Member, IEEE, \\ and Ricardo S. Sánchez-Peña, Senior Member, IEEE
}

\begin{abstract}
The article addresses the LPV control of a Polymer Electrolyte Membrane Fuel Cell (PEMFC). In order to optimize efficiency, PEMFCs require reliable control systems ensuring stability and performance, as well as robustness to model uncertainties and external perturbations. On the other hand, PEMFCs present a highly nonlinear behavior that demands nonlinear and/or adaptive control strategies to achieve high performance in the entire operating range. Here, a linear parameter varying (LPV) gain scheduled control is proposed. The control is based on a piecewise affine LPV representation of the PEMFC, a model that can be available in practice. In order to deal with the saturation of the control action, an LPV anti-windup compensation is also proposed. The complete control strategy is applied to several experimental practical situations in a laboratory fuel cell system to evaluate its performance and the reliability of the proposed algorithms.
\end{abstract}

Index Terms-Linear parameter varying systems; gain scheduled control; anti-windup; PEM fuel cells; oxygen stoichiometry.

\section{INTRODUCTION}

I NCREASING demands on pollution reduction are driving innovation on clean energy sources. Among these, fuel cells (FCs) are regarded as one of the most promising technologies, due to their potential efficiency, compactness and reliability [1]. Particularly, Polymer Electrolyte Membrane Fuel Cells (PEMFCs) are electrochemical devices that generate electrical energy from hydrogen and oxygen, with pure water and heat as only by-products. Considering that hydrogen is widely available and can be obtained from many renewable sources using solar and wind energy, FCs represent an attractive, feasible alternative to reduce fossil fuel dependence. However,

F.D. Bianchi is with Catalonia Institute for Energy Research, IREC, Jardins de les Dones de Negre 1, 08930 Sant Adrià de Besòs, Barcelona, Spain. email: fbianchi@irec.cat

C. Kunusch and C. Ocampo-Martinez are with Universitat Politècnica de Catalunya, Institut de Robòtica i Informàtica Industrial (CSIC-UPC), Llorens i Artigas, 4-6, 08028 Barcelona, Spain.

R.S. Sánchez-Peña is with CONICET and Instituto Tecnológico de Buenos Aires (ITBA), Av. Madero 399, (C1106ACD) Buenos Aires, Argentina.

The research of F.D. Bianchi was supported by the European Regional Development Funds (ERDF, FEDER Programa Competitivitat de Catalunya 2007-2013).

The research of C. Kunusch has been supported by the Seventh Framework Programme of the European Community through the Marie Curie actions (GA: PCIG09-GA-2011-293876) and project Puma-Mind (GA: FCH-JU-2011-1303419), as well as by the CICYT project DPI2011-25649 (MICINN-Spain).

The research of C. Ocampo-Martinez has been supported by the project MACPERCON (Ref. 201250E027) of the CSIC.

The research of R.S. Sánchez Peña has been supported by CONICET and grant PICT2008-290 from the PRH Program of the Ministry of Science, Technology and Innovation of Argentina. the widespread use of hydrogen as fuel -and the resulting "hydrogen economy"- despite its interesting possibilities, has some technological issues to be solved. In spite of recent advances, relatively high costs, suboptimal efficiency and reduced lifetime of FCs remain as major limitations [2], [3]. For instance, a cost analysis by [4] showed that a reduction of a FC plant investment costs $1,000 € / \mathrm{kW}$ could result in reduced generating costs compared with conventional devices as combustion engines. Clearly this depends on successful cost reduction as well as market rates for power and fuel. Present costs of fuels cells are as high as $5,000-10,000 € / \mathrm{kW}$, while possible future costs could down to $1,200-2,000 € / \mathrm{kW}$ for installation of a complete FC system [3].

For this reason, together with the continuous improvement of materials and components, the incorporation of advanced control strategies is essential in order to achieve cost reduction, performance improvement and efficiency optimization, as established in [5]. For instance, the net efficiency of a FC stack that runs with an air compressor directly depends on the proper regulation of certain variables such as stack oxygen flow and pressure. Here, reliable control laws should ensure the enhancement of not only the efficiency of the whole PEMFC system, but also its dynamic performance.

In the light of these considerations, it becomes clear that, in order to optimize efficiency, reliable control systems ensuring extended stability and performance, as well as robustness against model uncertainties and external perturbations are of critical importance for PEMFC success and future industrial developments [6], [7]. In particular, the oxygen stoichiometry control is a challenging problem [8], [9], [10], since it deals with system non-linearities and must be able to optimize the overall conversion efficiency in the entire operation range, avoiding performance deterioration together with eventual irreversible damages in the polymeric membranes due to oxygen starvation. Different control solutions for this problem have been proposed during the last few years, ranging from linear quadratic regulators (LQRs) [8], [11] to sliding mode based algorithms [6], [12]. In the latter works, the variable structure design solves the robust stability of the system, but lacks of a systematic way of developing controllers to enhance the dynamic performance.

As a novel solution to this technological problem, a robust oxygen stoichiometry control design and its implementation in a laboratory FC system are presented in this work. In particular, a linear parameter varying (LPV) gain scheduled control strategy based on a set of local models is designed and successfully tested experimentally. This produces a set 
of linear controllers interpolated in order to obtain the global control algorithm, an approach especially useful for complex systems in which analytic LPV models are difficult or impossible to obtain. Another contribution of this paper is an anti-windup (AW) compensation scheme for LPV controllers, a topic rarely analyzed in the literature. Several advantages of the LPV approach with respect to other techniques are here exploited for the considered problem in PEMFCs.

- Model uncertainty and bounded perturbations sets are naturally included and stability/performance guarantees are provided.

- The LPV approach considers the nonlinear nature of the plant besides providing robust stability and performance guarantees based on Linear Matrix Inequalities (LMI) convex optimization [13].

- The time-varying controller update is based solely on the variable measurements, which is critical due to the fast dynamics of this application. This fact provides a prompt response as opposed to adaptive control or model predictive control (MPC), which are based on real-time identification or optimization, respectively, more suitable for slower dynamics. Nevertheless, some approaches using explicit MPC controllers [14] or MPC emulators [15] are reported for real-time control of PEMFC. Other relevant works such as [16] propose explicit MPC controllers for LPV systems, guaranteeing constraint satisfaction, recursive feasibility and asymptotic stability in simulation. These approaches avoid online optimization, but significantly increase the complexity of the controller compared with LPV techniques.

Only few preliminary approaches have been reported in the literature regarding LPV on FCs. In fact, the use of LPV techniques for FCs is considered for performing control-oriented models [17], [18], [19], designing LPV-based controllers [19], and developing fault diagnosis methodologies based on LPV observers [20]. Related to FC modeling, in [17] the usefulness of the LPV model structure is explored for model reduction of a detailed physical model of a solid-oxide FC stack. In [19], an affine quasi-LPV model, based on identified models of the stack voltage and the air compressor flow, are used to design a LPV control for disturbance rejection and reference tracking, evaluated then by simulations. Notice that, in the best of the author's knowledge, these few references represent the current literature concerning the application of LPV techniques to FC based systems. None of them present experimental results of controller implementations, which in fact represent a very relevant contribution of this paper.

Notation: $\mathbb{R}(\mathbb{C})$ is the set of real (complex) numbers and $\mathbb{R}^{n \times m}$ the set of real matrices of $n \times m$. The Kronecker product is represented by $\otimes$. For a symmetric matrix $X \in \mathbb{R}^{n \times n}$, $X>0(X<0)$ denotes positive (negative) definiteness. Given symmetric matrices $X, Y$ and a general matrix $Z$, the following notation will be used $Z+Z^{T}=Z+(\star)$ and

$$
\left[\begin{array}{cc}
X & Z \\
Z^{T} & Y
\end{array}\right]=\left[\begin{array}{cc}
X & Z \\
\star & Y
\end{array}\right],
$$

where $\star$ completes the symmetric matrix.

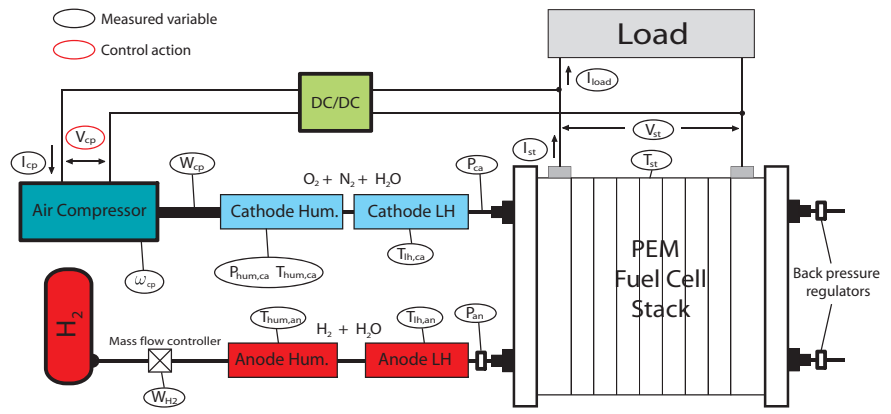

Fig. 1. Schematic diagram of the PEMFC based generation system (FCGS)

\section{SySteM PHYSICAL DESCRIPTION}

Concisely, the laboratory test plant under consideration mainly comprises a central PEMFC stack and ancillary units. A schematic diagram of the system is depicted in Fig. 1, and the main subsystems are briefly described below.

- Air Compressor: 12 V DC oil-free diaphragm vacuum pump. The input voltage $V_{c p}$ of this device is used as the control action.

- Hydrogen and oxygen humidifiers and line heaters: these are used to maintain proper humidity and temperature conditions inside the cell stack, an important issue for PEM membranes. Cellkraft ${ }^{\circledR}$ membrane exchange humidifiers are used in the current set-up. Decentralized PID controllers ensure adequate operation values.

- Fuel cell stack: an ZBT ${ }^{\circledR}$ 8-cell stack with Nafion $115^{\circledR}$ membrane electrode assemblies (MEAs) is used, $50 \mathrm{~cm}^{2}$ of active area and $150 \mathrm{~W}$ power.

In addition, to measure the required and further experimental data, different sensors are incorporated into the system: an air mass flow meter (range 0-15 slpm) at the end of the compressor to measure its flow $\left(W_{c p}\right)$, a current clamp (range 0-3 A) and a voltage meter (range 0-15 V) to measure the motor stator current $\left(I_{s t}\right)$ and voltage $\left(V_{c p}\right)$, respectively. Besides, temperature sensors are arranged in order to register the different operation conditions. For further details, refer to [6] where the most relevant components are characterized.

In the sequel, the following modeling assumptions have been considered [21], [6].

- A mass flow control device ensures a constant hydrogen stoichiometry supply.

- An auxiliary control system efficiently regulates gas temperatures at five points of the plant: cathode and anode humidifiers, cathode and anode line heaters and stack.

- A humidity control loop regulates the water injection of the humidifiers to a relative level close to $100 \%$.

- The FC model is one dimensional, so the gases and reactions are considered uniformly distributed in the cell.

- The electrochemical properties are evaluated at the average stack temperature $\left(70^{\circ} \mathrm{C}\right)$, so temperature variations across the stack are neglected.

- The water entering the cathode and anode is only in the vapor phase.

- The effects of liquid water creation are negligible at the gas flow model level. 
- The water activity is uniform across the membrane and is in equilibrium with the water activity at the cathode and anode catalyst layers.

The nonlinear model of the plant was already developed and validated in [21]. In general terms, the modeling process was conducted following a modular methodology, combining a theoretical approach, together with empirical analysis based on experimental data. Taking the state vector $\tilde{x} \in \mathbb{R}^{7}$ of the complete nonlinear model, the control input for the current study is the compressor voltage $v=V_{c p} \in \mathbb{R}$, the external disturbance is the stack current $I_{s t} \in \mathbb{R}$ and the output is the inlet stoichiometry $\lambda_{O_{2}} \in \mathbb{R}$. Accordingly, the system can be represented by the following continuous state-space equation

$$
\dot{\tilde{x}}(t)=f(\tilde{x}(t), t)+g(\tilde{x}) v(t),
$$

where $f: \mathbb{R}^{8} \rightarrow \mathbb{R}^{7}, g: \mathbb{R}^{7} \rightarrow \mathbb{R}^{7}$, and the state variables are defined as

- $\tilde{x}_{1}=\omega_{c p}$ : motor shaft angular velocity;

- $\tilde{x}_{2}=m_{\text {hum }, c a}$ : air mass inside the cathode humidifier;

- $\tilde{x}_{3}=m_{O_{2}, c a}$ : oxygen mass in the cathode channels;

- $\tilde{x}_{4}=m_{N_{2}, c a}$ : nitrogen mass in the cathode channels;

- $\tilde{x}_{5}=m_{v, c a}$ : vapor mass in the cathode channels;

- $\tilde{x}_{6}=m_{H_{2}, a n}$ : hydrogen mass in the anode channels;

- $\tilde{x}_{7}=m_{v, a n}$ : vapor mass in the anode channels.

It can be shown that the efficiency optimization of the current system can be achieved by regulating the oxygen mass inflow towards the stack cathode [6]. If an adequate oxidant flow is ensured through the stack, the load demand is satisfied with minimum fuel consumption. Additionally, oxygen starvation and irreversible membrane damage are averted. For more details please refer to [8] and [6], where the concepts of FCs net efficiency and their relationship with the oxidant flow are presented.

To accomplish such an oxidant flow is equivalent to maintain the oxygen excess ratio of the cathode at a suitable value. The oxygen excess ratio or oxygen stoichiometry is defined as

$$
\lambda_{O_{2}}(t)=\frac{W_{O_{2}, c a}(t)}{W_{O_{2}, \text { react }}(t)},
$$

where $W_{\mathrm{O}_{2} \text {, react }}(t)$ is the oxygen flow consumed in the reaction and $W_{O_{2}, c a}(t)$ is the oxygen partial flow entering the cathode, which depends on the air flow released by the compressor $W_{c p}(t)$, i.e.,

$$
W_{O_{2}, c a}(t)=\frac{\chi_{O_{2}} W_{c p}(t)}{1+\omega_{a m b}(t)}
$$

being $\omega_{a m b}(t)$ the ambient air humidity ratio and $\chi_{O_{2}}$ the molar fraction of oxygen in the air $\left(\chi_{\mathrm{O}_{2}}=0.21\right)$.

Notice that $W_{O_{2}}$,react $(t)$ is directly related to the stack current in the form

$$
W_{O_{2}, \text { react }}(t)=G_{O_{2}} \frac{n I_{s t}(t)}{4 F},
$$

with $G_{\mathrm{O}_{2}}$ the molar mass of oxygen, $n$ the number of cells and Faraday's constant $F$.

As presented in the validated model [21], the operating conditions of the system inputs are determined by $V_{c p}$ and $I_{s t}$. In this context, these two variables represent a natural selection to parameterize the nonlinear system in terms of an LPV model. In the following, it will be assumed that the parameter space belongs to $\mathbb{R}^{2}$.

The control objective considered in this article is to track the oxygen stoichiometry $\lambda_{O_{2}}(t)$ such that

$$
\lim _{t \rightarrow \infty}\left(\lambda_{O_{2}}(t)-\lambda_{O_{2}, \text { ref }}\right)=0,
$$

where $\lambda_{O_{2} \text {, ref }}$ is a given reference, under continuous changes in the load conditions $\left(I_{s t}\right)$.

\section{LPV CONTROL OF PEM FUEL CELLS}

LPV system theory has arisen as an elegant formulation of the widely spread gain-scheduling techniques. The LPV formulation provides synthesis tools that guarantee stability and performance of the closed-loop system in all operating conditions considered in the design. However, to obtain these guarantees, analytic expressions to describe the behavior of the nonlinear system are necessary. This is not always possible, especially in cases of complex models based on look-up table parameters or with very complex mathematical expressions. In many of these cases, such as the PEMFC based system (1), only a set of linear models describing the local behavior at a set of operating points are available. With no additional information, a linear interpolation of the model matrices corresponding to the closest points is commonly used to describe the system behavior at intermediate points. This approximation works in practice if the grid is dense enough and/or the small errors are covered by uncertainty. This kind of LPV systems, known as piecewise affine LPV (PALPV) systems [22], will be described in the next subsection along with the synthesis procedure. Afterwards, an AW compensation is proposed and finally some comments concerning the discretization of the controller are provided.

\section{A. LPV description and control design}

The LPV description starts with the definition of the parameter space, which is assumed in $\mathbb{R}^{2}$ since the PEMFC model can be parameterized with two parameters.

Let $\theta \in \mathbb{R}^{2}$ be a parameter taking values in a polytope $\Theta=\left[\underline{\theta}_{1}, \bar{\theta}_{1}\right] \times\left[\underline{\theta}_{2}, \bar{\theta}_{2}\right]$. Assume that the parameter set is partitioned into $\left(m_{1}-1\right) \times\left(m_{2}-1\right)$ closed rectangles $\Theta_{i j}$ 's. Then, the parameter $\theta$ can be expressed as

$$
\theta(t)=\sum_{i=1}^{m_{1}} \sum_{j=1}^{m_{2}} \alpha_{i j}(t) \hat{\theta}_{i j}
$$

where $\hat{\theta}_{i j}$ are the points on a grid

$$
\mathcal{G}=\left\{\hat{\theta}_{i j}, i=1, \ldots, m_{1}, j=1, \ldots, m_{2}\right\},
$$

defining the partition of the rectangles $\Theta_{i j}$ 's,

$$
\sum_{i=1}^{m_{1}} \sum_{j=1}^{m_{2}} \alpha_{i j}=1
$$

with

$$
\alpha_{i j}=\psi\left(\frac{\hat{\theta}_{(i+1)(j+1), 1}-\theta_{1}}{\hat{\theta}_{(i+1)(j+1), 1}-\hat{\theta}_{i j, 1}} \cdot \frac{\hat{\theta}_{(i+1)(j+1), 2}-\theta_{2}}{\hat{\theta}_{(i+1)(j+1), 2}-\hat{\theta}_{i j, 2}}\right)
$$


and

$$
\psi(\vartheta)= \begin{cases}\vartheta, & \text { if } 0<\vartheta \leq 1 \\ 0, & \text { otherwise. }\end{cases}
$$

That is, if $\theta \in \Theta_{i j}$, then $\theta$ is expressed as a convex combination of the vertices of the rectangle $\Theta_{i j}$,

$$
\begin{aligned}
\theta=\alpha_{i j} \theta_{i j}+\alpha_{i(j+1)} \theta_{i(j+1)}+ & \alpha_{(i+1) j} \theta_{(i+1) j}+ \\
& +\alpha_{(i+1)(j+1)} \theta_{(i+1)(j+1)} .
\end{aligned}
$$

Then, the system's description is assumed as

$$
\left[\begin{array}{c}
\dot{x}(t) \\
z(t) \\
e(t)
\end{array}\right]=\sum_{i=1}^{m_{1}} \sum_{j=1}^{m_{2}} \alpha_{i j}(t)\left[\begin{array}{ccc}
A_{i j} & B_{1, i j} & B_{2} \\
C_{1, i j} & D_{11, i j} & D_{12} \\
C_{2} & D_{21} & 0
\end{array}\right]\left[\begin{array}{c}
x(t) \\
w(t) \\
u(t)
\end{array}\right],
$$

being $x \in \mathbb{R}^{n_{s}}$ the state, $z \in \mathbb{R}^{n_{z}}$ a performance output, $y \in \mathbb{R}^{n_{y}}$ the measured variable, $w \in \mathbb{R}^{n_{w}}$ the disturbance and $u \in \mathbb{R}^{n_{u}}$ the control input. Expression (10) describes the system as an affine LPV model in each rectangle $\Theta_{i j}$ and the matrices are piecewise continuous functions of the parameter $\theta$.

With the previous assumption, the gain-scheduled controller

$$
\left[\begin{array}{c}
\dot{x}_{c}(t) \\
u(t)
\end{array}\right]=\sum_{i=1}^{m_{1}} \sum_{j=1}^{m_{2}} \alpha_{i j}(t)\left[\begin{array}{cc}
A_{c, i j} & B_{c, i j} \\
C_{c, i j} & D_{c, i j}
\end{array}\right]\left[\begin{array}{c}
x_{c}(t) \\
e(t)
\end{array}\right]
$$

should guarantee that the induced $\mathcal{L}_{2}$ norm of the operator $T_{z w}: w \rightarrow z$, mapping the disturbance $w$ to the output $z$, satisfies

$$
\left\|T_{z w}\right\|_{\mathcal{L}_{2}}=\sup _{w \neq 0, \theta \in \Theta} \frac{\|z\|_{2}}{\|w\|_{2}}<\gamma
$$

and the local closed-loop systems have all their poles in an LMI region

$$
\mathcal{D}=\left\{s \in \mathbb{C} \mid \Gamma+s \Upsilon+\bar{s} \Upsilon^{T}<0\right\},
$$

where $\bar{s}$ is the conjugate of $s, \Gamma$ and $\Upsilon$ are real matrices of suitable dimensions and " $<$ " stands for negative definite (see [23] for more details). For example, the LMI region

$$
\mathcal{D}=\{s \in \mathbb{C}|-\beta \leq \operatorname{Re}(s)<0 \cap| \operatorname{Im}(s) \mid<\beta\}
$$

can be described in the form (13) with matrices

$$
\Gamma=\left[\begin{array}{ccc}
-2 \beta & 0 & 0 \\
0 & -\beta & 0 \\
0 & 0 & -\beta
\end{array}\right], \quad \Upsilon=\left[\begin{array}{ccc}
-1 & 0 & 0 \\
0 & 0 & -1 \\
0 & 1 & 0
\end{array}\right] .
$$

The LPV controller (11) satisfying conditions (12) and (13) is computed by finding two symmetric positive definite matrices $X$ and $Y$ and matrices $\hat{A}_{c, i j}, \hat{B}_{c, i j}, \hat{C}_{c, i j}$ and $D_{c, i j}$ such that the LMI conditions (15)-(16) and

$$
\left[\begin{array}{cc}
X & I \\
I & Y
\end{array}\right]>0
$$

are satisfied for all $i=1, \ldots, m_{1}$ and $j=1, \ldots, m_{2}$. The controller matrices are given by

$$
\begin{gathered}
A_{c, i j}=N^{-1}\left(\hat{A}_{c, i j}-X\left(A_{i j}-B_{2} D_{c, i j} C_{2}\right) Y-\hat{B}_{c, i j} C_{2} Y-\right. \\
\left.-X B_{2} \hat{C}_{c, i j}\right) M^{-T}, \\
B_{c, i j}=N^{-1}\left(\hat{B}_{c, i j}-X B_{2} D_{c, i j}\right), \\
C_{c, i j}=\left(\hat{C}_{c, i j}-D_{c, i j} C_{2} Y\right) M^{-T},
\end{gathered}
$$

where $I-X Y=N M^{T}$ [24].

Notice that the previous formulation is based on a constant Lyapunov function, unlike piecewise discontinuous [22] or continuous parameter dependent Lyapunov functions [25], [24] commonly used in PALPV approaches. Although a constant Lyapunov function may result conservative, this approach is used to simplify the implementation of the controller in an industrial computer. As will be seen in Section IV, this approach produces suitable results in the present application.

In the case of the PEMFC, the LPV description (10) is obtained from the nonlinear model in (1) by linearizing around a set of operating points defined by the mean values of the compressor voltage and stack current, $V_{c p, 0}$ and $I_{s t, 0}$ respectively. Thus, the linear models are parameterized by $\theta=\left[\begin{array}{lll}V_{c p, 0} & I_{s t, 0}\end{array}\right]^{T}$. The Taylor expansion of (1) around each $\hat{\theta}_{i j} \in \mathcal{G}$ is defined as follows

$G_{i j}:\left\{\begin{array}{l}\dot{x}(t)=\left[\frac{\partial f(x(t), t)}{\partial x(t)}\right]_{\hat{\theta}_{i j}} x(t)+\left[\frac{\partial g(x(t))}{\partial x(t)}\right]_{\hat{\theta}_{i j}} u(t), \\ y(t)=\left[\begin{array}{lllllll}1 & 0 & 0 & 0 & 0 & 0 & 0\end{array}\right] x(t),\end{array}\right.$

where $x(t)=\tilde{x}(t)-x_{0}\left(\hat{\theta}_{i j}\right), u(t)=v(t)-v_{0}\left(\hat{\theta}_{i j}\right)$. To simplify the notation, the PALPV description obtained from the local systems (17) will be hereafter referred as $G(\theta)$ and the inputoutput mapping as

$$
y(t)=G(\theta) * u(t)
$$

In order to design the controller as previously described, it is necessary to define the augmented plant (10). That is, the interconnection between the nominal model and the design weights with the disturbance and controller output $\left[\begin{array}{ll}w & u]^{T} \text { as }\end{array}\right.$ inputs and the performance signal and controller input $[z e]^{T}$ as outputs. In the case of the PEMFC problem, the design can be expressed as a typical mixed sensitivity problem. The main objective is to maintain the oxygen stoichiometry $\lambda_{O_{2}}$ close to the reference value $\lambda_{O_{2} \text {, ref }}$ (i.e., $e=\lambda_{O_{2} \text {, ref }}-\lambda_{O_{2}}$ close to 0 ), in addition to keep limited the control action

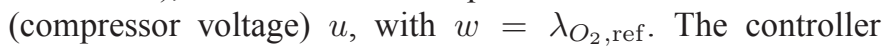
output is saturated to ensure $V_{c p}$ never exceeds the maximum and minimum levels. Therefore, the performance signal is given by $z=[\tilde{e} \tilde{u}]^{T}$, where tilde $\sim$ denotes the weighted version of these signals.

The augmented plant is shown in Fig. 2, where $\tilde{K}(\theta)$ is the controller (11) produced by the synthesis procedure previously described. Integral action is included to ensure zero steadystate error. Thus, the weighting function $W_{e}$, which can be a simple constant, penalizes the error in low frequencies to guarantee the system operates at the desired set-point. On the other hand, the weight $W_{u}$ penalizes the high frequency components of the control action. This weighting function also allows considering the model (additive) uncertainty associated with the differences caused by the interpolation assumption and the theoretical model. The complete controller is thus given by $K(\theta)=(1 / s) \cdot \tilde{K}(\theta)$, where $\cdot$ stands for the series interconnection of two systems. 


$$
\begin{aligned}
& {\left[\begin{array}{cccc}
X A_{i j}+\hat{B}_{c, i j} C_{2}+(\star) & \star & \star & \star \\
\hat{A}_{c, i j}^{T}+A_{i j}+B_{2} D_{c, i j} C_{2} & A_{i j} Y+B_{2} \hat{C}_{c, i j}+(\star) & \star & \star \\
\left(X B_{1, i j}+\hat{B}_{c, i j} D_{21}\right)^{T} & \left(B_{1, i j}+B_{2} D_{c, i j} D_{21}\right)^{T} & -\gamma I_{n_{w}} & \star \\
C_{1, i j}+D_{12} D_{c, i j} C_{2} & C_{1, i j} Y+D_{12} \hat{C}_{c, i j} & D_{11, i j}+D_{12} \hat{D}_{c, i j} D_{21} & -\gamma I_{n_{z}}
\end{array}\right]<0,} \\
& \Gamma \otimes\left[\begin{array}{cc}
X & I \\
I & Y
\end{array}\right]+\Upsilon^{T} \otimes\left[\begin{array}{ccc}
X A_{i j}+\hat{B}_{c, i j} C_{2} & \hat{A}_{c, i j} \\
A_{i j}+B_{2} D_{c, i j} C_{2} & A_{i j} Y+B_{2} \hat{C}_{c, i j}
\end{array}\right]+(\star)<0 .
\end{aligned}
$$

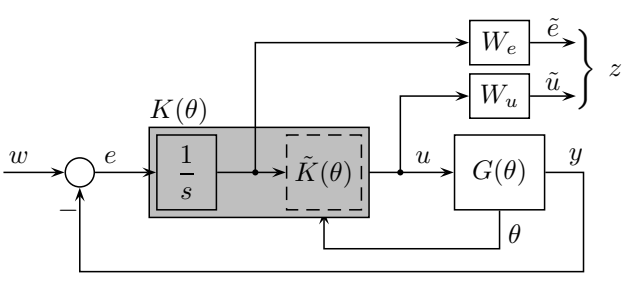

Fig. 2. Plant augmented with weighting functions

\section{B. Anti-windup compensation}

As mentioned, the compressor voltage must be saturated to avoid exceeding the operating limits. To prevent the undesirable effects of the saturation of the control action, an AW compensation is added to the LPV controller (11).

There are a large number of AW compensation schemes in the literature (see [26] and references therein). However, only a few articles address the problem in case of LPV systems [27], [28], [29]. Using the general framework introduced in [30], an AW compensation scheme is proposed to fit the system definition given in the previous subsection.

The AW compensation scheme is sketched in Fig. 3. It consists of two compensation terms: one acting on the controller output $u_{d}$ and another on the controller input $y_{d}$. Defining

$$
\left[\begin{array}{l}
u_{d} \\
y_{d}
\end{array}\right]=T_{a w}(\theta) * \check{u}=\left[\begin{array}{c}
M(\theta)-I \\
N(\theta)
\end{array}\right] * \check{u},
$$

where $N(\theta)=G(\theta) \cdot M(\theta)$, it can be proved, after some system manipulations, that the compensation scheme in Fig. 3 reduces to the equivalent scheme in Fig. 4. From this figure, it can be seen that $M(\theta)$ must be designed to ensure stability of the closed-loop system formed by $M(\theta)-I$ and the nonlinear operator and to minimize the effect of the $y_{d}$ on the controlled variable. Moreover, applying the LPV coprime factorization introduced in [31] to the LPV system $G(\theta)$, the design of the AW compensator reduces to the design of a parameter-varying state-feedback gain fulfilling an induced $\mathcal{L}_{2}$ norm condition.

More precisely, let

$$
\left[\begin{array}{c}
\dot{x}_{a w}(t) \\
u_{d}(t) \\
y_{d}(t)
\end{array}\right]=\sum_{i=1}^{m_{1}} \sum_{j=1}^{m_{2}} \alpha_{i j}(t)\left[\begin{array}{cc}
A_{i j}+B_{2} H_{i j} & B_{2} \\
H_{i j} & 0 \\
C_{2} & 0
\end{array}\right]\left[\begin{array}{c}
x_{a w}(t) \\
\check{u}(t)
\end{array}\right]
$$

be the state-space realization of $T_{a w}(\theta)$, where $H$ is a statefeedback gain such that $T_{a w}(\theta)$ is quadratically stable for $\theta \in$ $\Theta$. Then, using the Small Gain Theorem, the AW compensator will ensure quadratic stability during saturation if $\| M(\theta)-$ $I \|_{\mathcal{L}_{2}}<1$. The minimization of the effect on the controlled variable can similarly be expressed as $\|N(\theta)\|_{\mathcal{L}_{2}}<\nu$. Both

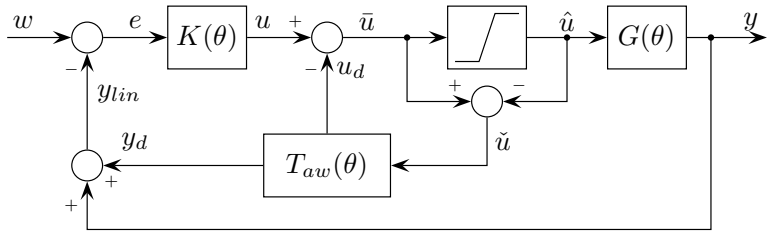

Fig. 3. Anti-windup compensation scheme

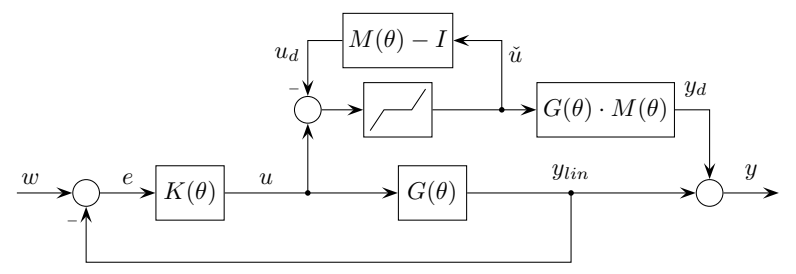

Fig. 4. Equivalent representation of the anti-windup compensation scheme in Fig. 3

conditions will be satisfied if

$$
\left\|\begin{array}{c}
M(\theta)-I \\
N(\theta)
\end{array}\right\|_{\mathcal{L}_{2}}<\nu
$$

with $\nu<1$. Therefore, using standard results from LPV theory [32], [33], the following can be proved.

Theorem 3.1: There exists a AW compensator $T_{a w}(\theta)$ that ensures stability and minimizes the effect of the actuator saturation on the controlled output in the sense of $\mathcal{L}_{2}$ (condition (18)) if there exist matrices $Q^{T}=Q>0, W_{i j}$ and a positive scalar $\nu<1$ such that the following LMIs are satisfied

$$
\left[\begin{array}{cccc}
A_{i j} Q+B_{2} W_{i j}+(\star) & \star & \star & \star \\
B_{2}^{T} & -\nu I_{n_{u}} & \star & \star \\
W_{i j} & I & -\nu I_{n_{u}} & \star \\
C_{2} Q & 0 & 0 & -\nu I_{n_{y}}
\end{array}\right]<0
$$

for all $i=1, \ldots, m_{1}, j=1, \ldots, m_{2}$, and $H_{i j}=Q^{-1} W_{i j}$.

The controller with the AW compensation is given by

$$
\left[\begin{array}{c}
\dot{x}_{c}(t) \\
u(t)
\end{array}\right]=\sum_{i=1}^{m_{1}} \sum_{j=1}^{m_{2}} \alpha_{i j}(t)\left[\begin{array}{ccc}
\bar{A}_{c, i j} & \bar{B}_{c 1, i j} & \bar{B}_{c 2} \\
\bar{C}_{c, i j} & D_{c, i j} & 0
\end{array}\right]\left[\begin{array}{c}
x_{c}(t) \\
e(t) \\
\check{u}(t)
\end{array}\right]
$$

where

$$
\begin{aligned}
\bar{A}_{c, i j} & =\left[\begin{array}{cc}
A_{c, i j} & -B_{c 1, i j} C_{2} \\
0 & A_{i j}+B_{2} H_{i j}
\end{array}\right], \quad \bar{B}_{c 1, i j}=\left[\begin{array}{c}
B_{c 1, i j} \\
0
\end{array}\right]^{T}, \\
\bar{B}_{c 2} & =\left[\begin{array}{ll}
0 & B_{2}^{T}
\end{array}\right]^{T}, \quad \bar{C}_{c 1, i j}=\left[\begin{array}{ll}
C_{c, i j} & -\left(D_{c, i j} C_{2}+H_{i j}\right)
\end{array}\right] .
\end{aligned}
$$

Notice that the controller with AW compensation preserves the parameter dependence of the original controller. This is a consequence of using a single quadratic Lyapunov function. 
Although this approach could be conservative in general applications, it simplifies the implementation without sacrificing the performance in the PEMFC case. Notice also that the AW compensation only depends on the non saturated system $G(\theta)$.

The LMIs (19) in Theorem 3.1 can be complemented with the pole placement constraints

$$
\Gamma \otimes Q+\Upsilon^{T} \otimes\left(A_{i j} Q+B_{2} W_{i j}\right)+(\star)<0,
$$

for all $i=1, \ldots, m_{1}$ and $j=1, \ldots, m_{2}$. These additional constraints ensure that the complete controller (20) can be implemented in discrete time with the desired sampling time.

\section{Discrete implementation}

Finally, the continuous-time controller (20) must be translated into a discrete-time system before its implementation. This is not a trivial task in LPV systems because discretization changes the state-space realizations and thus the parameter dependence of the LPV controller. To preserve the affine dependence, the system is discretized with Euler's forward method and the following approximation

$$
e^{T_{s} A_{c}\left(\theta\left(k T_{s}\right)\right)} \simeq I+T_{s} A_{c}\left(\theta\left(k T_{s}\right)\right),
$$

where $T_{s}$ is the sampling time [34]. With this approximation, the controller in (20) becomes

$$
\left[\begin{array}{c}
\dot{x}_{c}(k) \\
u(k)
\end{array}\right]=\sum_{i=1}^{m_{1}} \sum_{j=1}^{m_{2}} \alpha_{i j}(k)\left[\begin{array}{ccc}
\bar{A}_{d, i j} & T_{s} \bar{B}_{c 1, i j} & T_{s} \bar{B}_{c 2} \\
C_{c, i j} & D_{c, i j} & 0
\end{array}\right]\left[\begin{array}{c}
x_{c}(k) \\
e(k) \\
\check{u}(k)
\end{array}\right]
$$

in discrete time, with $\bar{A}_{d, i j}=I+T_{s} \bar{A}_{c, i j}$.

\section{EXPERIMENTAL RESULTS}

Considering the LPV description of the system introduced in Section III-A, the gain scheduled controller previously described was designed in a grid of 16 operating points given by

$$
\mathcal{G}=\mathbf{V}_{c p, 0} \times \mathbf{I}_{s t, 0},
$$

with $\mathbf{V}_{c p, 0} \in\{3,6,9,12\} \mathrm{V}$ and $\mathbf{I}_{s t, 0} \in\{2,4,6,8\}$ A. This parameter grid results after reaching a compromise between implementation complexity and model accuracy. The number of points in the grid $\mathcal{G}$ is limited by the use of memory in the industrial computer. Recall that the number of matrices to be stored in memory by the control algorithm depends on the number of points in the grid. The modeling errors in the intermediate points, caused by the low grid density, were covered by additive uncertainty and taken into account during the design by selecting a proper weighting function $W_{u}$. As will be seen in the experimental results, the grid selection is suitable to describe the nonlinear system.

The weighting functions in the synthesis were selected as

$$
W_{e}(s)=0.5, \quad W_{u}(s)=0.005 \frac{s / 0.3+1}{s / 30+1} .
$$

The latter function penalizes the control action in high frequencies and also provides robustness against differences between the model and the actual system. The AW compensation was designed based on Theorem 3.1, after the gain scheduled controller (11) was computed. The controller and the AW compensator were designed with the LMI pole placement region (14) with $\beta=0.1 / T_{s}$, where $T_{s}=10 \mathrm{~ms}$. The optimization problems for obtaining the LPV controller and the AW compensation were solved using Sedumi [35] and Yalmip [36].

The complete control strategy was implemented in the data acquisition \& control system. It is composed of two computers (each with four cores i5 processor at $2.6 \mathrm{GHz}$ clock frequency): the host and the real-time operating system (RTOS). The host provides the software development environment and the graphical user interface. It is responsible for the start up, shut down, configuration changes and control settings during operation. The RTOS implements the control algorithms and the data acquisition via a fieldprogrammable gate array (FPGA), in order to have high speed data processing. Control, security and monitoring tasks are conducted by a CompactRIO (reconfigurable Input/Output) system from National Instruments. The LPV controller and the AW algorithm where developed in Matlab ${ }^{\circledR}$ and then cross-compiled into a LabView ${ }^{\circledR}$ environment. In order to record the analog sensor signals, a 32-channel 16-bit analog input module from National Instruments is used (NI-9205). An 8-channel, digital input/output (I/O) module generates the necessary transistor-transistor logic (TTL) signals for different security and diagnostic tools.

In order to evaluate the performance of the proposed LPV controller and the AW loop, four realistic scenarios were considered covering different working conditions and external disturbances.

a) Scenario 1: Here, the stack current $I_{s t}$ is kept constant at $6 \mathrm{~A}$ and different values are set for the reference of the

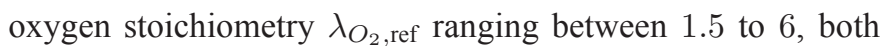
smoothly increasing and decreasing its value and performing abrupt changes in its magnitude. This is a typical scenario where the oxygen stoichiometry of a PEMFC based system is changed in order to obtain different net powers. Fig. 5 shows the response of the system under the aforementioned conditions. The figure also illustrates the behavior of the compressor flow $W_{c p}$ and how it varies according to the value of $\lambda_{\mathrm{O}_{2}}$. It is also shown how the oxygen stoichiometry tracking is suitably reached except for values of $\lambda_{O_{2}}$,ref greater than approximately 5.5 , since the control signal $V_{c p}$ is saturated. The control action saturation limit here is $12.4 \mathrm{~V}$. It is also important to highlight that there are no peaks in the transient of

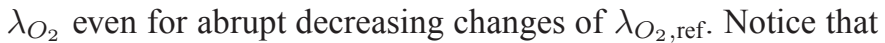
values of $\lambda_{\mathrm{O}_{2}}$ below one will cause irreversible damages in the FC stack. A natural step forward would be the incorporation of an extremum seeking algorithm to drive $\lambda_{O_{2} \text {,ref }}$ to its optimal value, usually between 1.5 and 3 .

b) Scenario 2: In an actual application, once the desired optimal value of $\lambda_{\mathrm{O}_{2}}$,ref is reached, it is interesting to evaluate the regulation behavior of the control system when current changes take place. To reproduce this typical working case, $\lambda_{\mathrm{O}_{2} \text {, ref }}$ was set constant at 2 , then different values of $I_{s t}$ were required from the PEMFC system. From this scenario, Fig. 6 shows the behavior of the related variables. Here, several

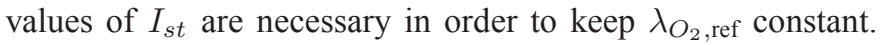



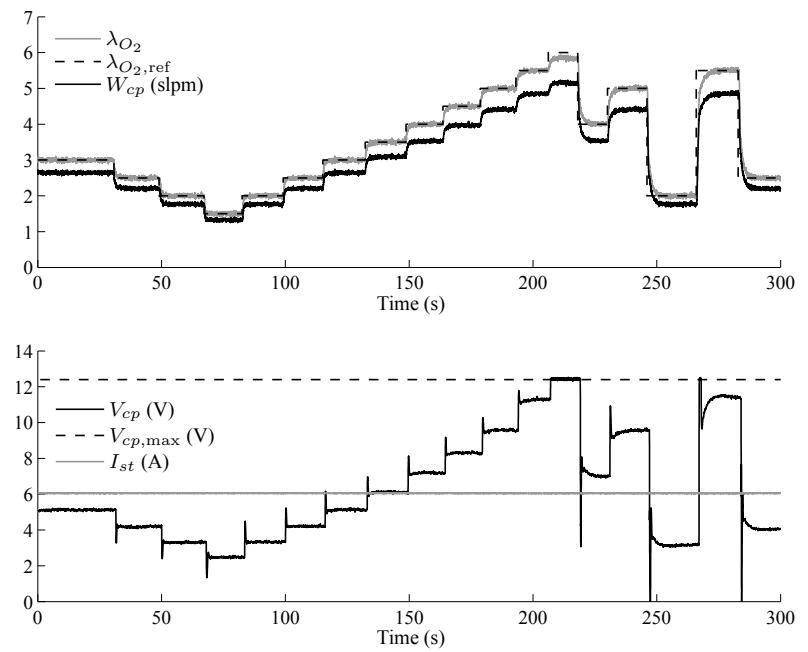

Fig. 5. Main variables related to test performed for Scenario 1.
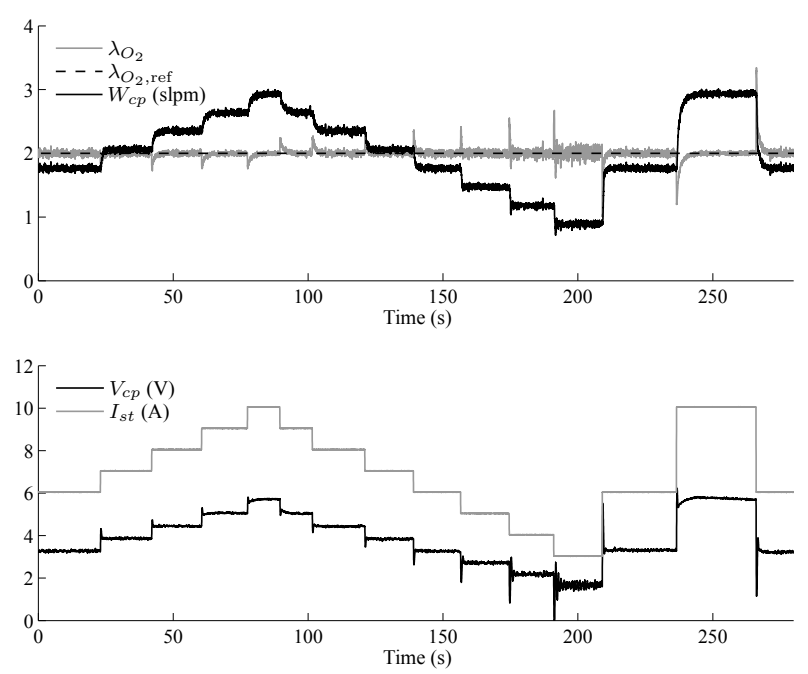

Fig. 6. Main variables related to test performed for Scenario 2.

Note the suitable regulation even for abrupt changes in $I_{s t}$ and for values of this current that were not taken into account neither in the model linearization nor in the controller design stage $\left(I_{s t}=10 \mathrm{~A}\right.$ from 240 to $265 \mathrm{~s}$, approximately). This shows the robustness of the proposed control strategy. The noisy behavior of $\lambda_{O_{2}}(t)$ around 200 s (i.e., for $I_{s t}=3 \mathrm{~A}$ ) is due to the small value of the compressor flow, which is given in turn, by the voltage $V_{c p}$ (control signal). Again, no peaks of $\lambda_{\mathrm{O}_{2}}$ below one were present despite the changes in $I_{s t}$.

c) Scenario 3: Here, the saturation of the compressor voltage $V_{c p}$ is induced by performing the regulation of $\lambda_{\mathrm{O}_{2}}$ at high levels (i.e., $\lambda_{O_{2}} \geq 5$ ) and considering $I_{s t} \in\{6,7\}$ A. In this realistic example, the system was tested under an actuator saturation situation. Fig. 7 illustrates what happens when the control action reaches the hardware limit $(12.4 \mathrm{~V})$. These abnormal situations can be seen during the intervals between 48 and $75 \mathrm{~s}$ and between 120 and $144 \mathrm{~s}$, approximately. Note that the AW algorithm increases the capacity of the controller to quickly recover without overshoots.

d) Scenario 4: Having verified the control operation in the nominal operation range, the system was tested under
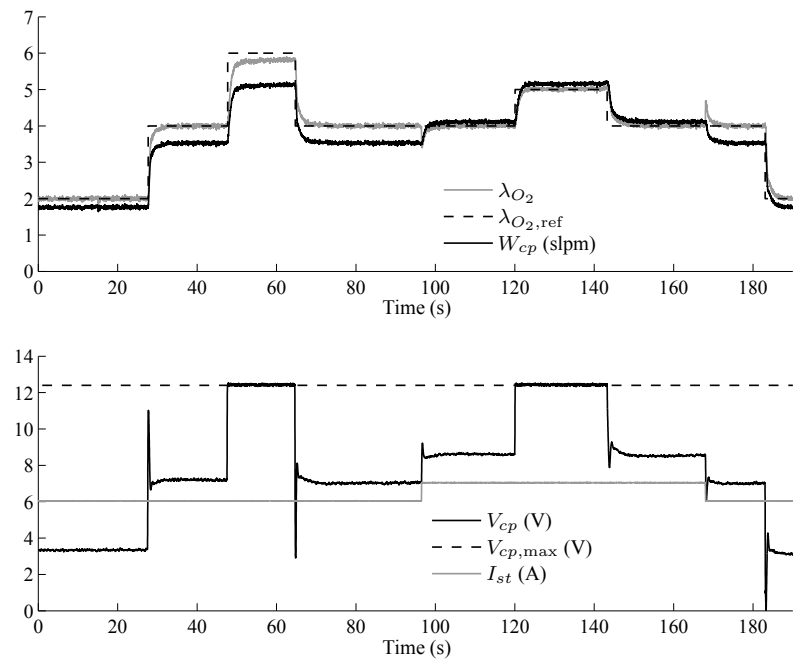

Fig. 7. Main variables related to test performed for Scenario 3.
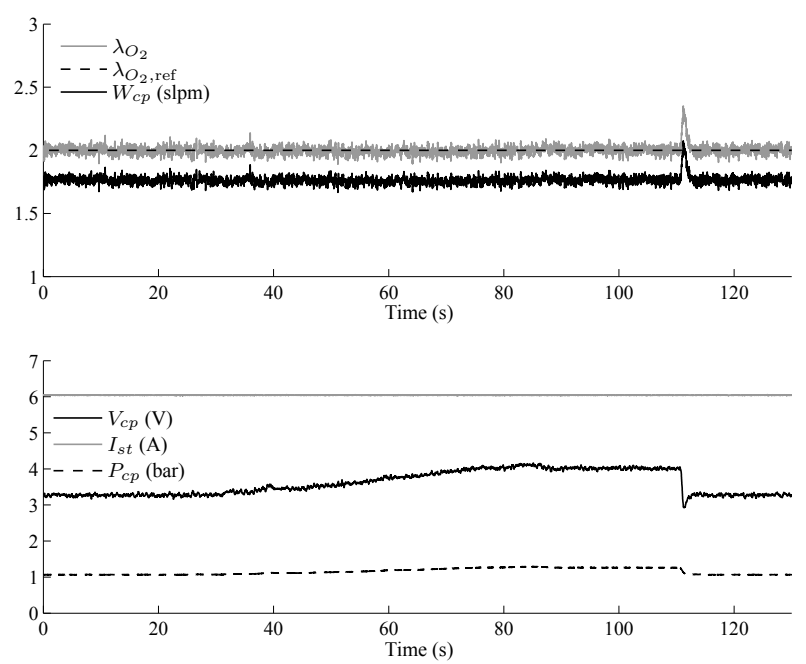

Fig. 8. Main variables related to test performed for Scenario 4.

the influence of external perturbations. This case may occur in practice when the cathode return manifold is throttled or an electronic valve is acting to keep constant the pressure between cathode and anode. In this particular test, $I_{s t}$ was kept constant at $6 \mathrm{~A}$ and an increment in the cathode's pressure $P_{c p}$ was forced using a mechanical back pressure regulator (from 1.1 bar to 1.3 bar). This effect can be appreciated in Fig. 8, where it is shown that from 20 to $110 \mathrm{~s}$, while the valve is increasingly throttled, the reference tracking is successfully preserved. Next, when the valve is suddenly bypassed ( $t=110 \mathrm{~s}$ ), the system output departs from the reference but the LPV controller provides a quick recovery.

The results presented in this section can be compared with the ones obtained by some of the authors using the same plant. In [6], a Super-Twisting algorithm has been developed to ensure robust stability of the system and a feedforward loop has been added to enhance the dynamic performance. The behavior of both controllers is comparable, even when disturbances arise, but it is important to stress that the LPV controller does not include a feedforward loop, making its design more systematic and robust against static uncertainty 
despite that its computational burden is slightly increased. On the other hand, in [11], an LQR controller has been experimentally tested considering the same plant and control objectives. Results show a suitable response when the system is close to the considered operating point, but its performance cannot be theoretically guaranteed in other working conditions.

\section{Conclusions}

An LPV gain scheduled control strategy has been proposed to regulate the oxygen stoichiometry of a PEMFC. A precise control of this variable is needed to ensure an efficient conversion and avoid irreversible damages in the polymeric membrane. Special attention has been paid to the implementation aspects. To this end, an LPV AW compensation has been introduced in order to mitigate the negative effects of the saturation of the control action. In addition, both in the LPV controller and in the AW compensator, pole placement constraints have been considered to guarantee a proper implementation in industrial computers. The complete control strategy has been implemented in an experimental platform and evaluated in several practical scenarios. In all cases, the proposed control has exhibited promising results.

\section{ACKNOWLEDGEMENTS}

All the experimental tests were performed at the Fuel Cells Laboratory of the Institut de Robòtica i Informàtica Industrial (CSIC-UPC, Barcelona, Spain) and only possible due to its advanced equipment and proficient technical staff.

\section{REFERENCES}

[1] "Department of Energy. Hydrogen, Fuel Cells And Infrastructure Technologies Program: Multi-Year Research, Development And Demonstration Plan: planned program activities for 2005-2015. DOE Website. Section 3.4 Fuel Cells.” Tech. Rep., 2005.

[2] F. Barbir, PEM Fuel Cells: Theory and Practice. Burlington, USA: Academic Press, 2005.

[3] J. Halliday, A. Ruddell, J. Powell, and M. Peters, "Fuel cells: providing heat and power in the urban environment," Tyndall Centre for Climate Change Research, Tech. Rep. 32, 2005.

[4] A. Lokurlu, T. Grube, B. Hohlein, and D. Stolten, "Fuel cells for mobile and stationary applications: Cost analysis for combined heat and power stations on the basis of fuel cells," Int. J. Hydrogen Energy, vol. 28, pp. 703-711, 2003.

[5] Fuel Cells and Hydrogen Joint Undertaking (FCH JU), European Union, 2013, guide to Financial Issues for beneficiaries of FCH JU Programme.

[6] C. Kunusch, P. Puleston, and M. Mayosky, Sliding-Mode Control of PEM Fuel Cells. London, UK: Springer London Ltd, 2012.

[7] Y. Tang, W. Yuan, M. Pan, Z. Li, G. Chen, and Y. Li, "Experimental investigation of dynamic performance and transient responses of a $\mathrm{kW}$ class PEM fuel cell stack under various load changes," Appl. Energ., vol. 87, no. 4, pp. 1410-1417, 2010.

[8] J. Pukrushpan, A. Stefanopoulou, and H. Peng, Control of Fuel Cell Power Systems. London, UK: Springer, 2004.

[9] J. Larminie and A. Dicks, Fuel Cell Systems Explained, 2nd ed. Chichester, UK: John Wiley \& Sons Ltd, 2003.

[10] J. Gruber, C. Bordons, and A. Oliva, "Nonlinear MPC for the airflow in a PEM fuel cell using a volterra series model," Control Eng. Pract., vol. 20, no. 2, pp. 205-217, 2012.

[11] A. Niknezhadi, M. Allué-Fantova, C. Kunusch, and C. OcampoMartinez, "Design and implementation of LQR/LQG strategies for oxygen stoichiometry control in PEM fuel cells based systems," J. Power Sources, vol. 196, no. 9, pp. 4277-4282, 2011.

[12] C. Kunusch, P. Puleston, M. Mayosky, and L. Fridman, "Experimental results applying second order sliding mode control to a pem fuel cell based system," Control Eng. Pract., vol. 21, no. 5, pp. 719-726, 2013.
[13] S. Boyd, L. El Ghaoui, E. Feron, and V. Balakrishnan, Linear Matrix Inequalities in System and Control Theory. New York, USA: SIAM Studies in Applied Mathematics, 1994.

[14] A. Arce, A. del Real, C. Bordons, and D. Ramirez, "Real-time implementation of a constrained mpc for efficient airflow control in a pem fuel cell," IEEE Trans. Ind. Electron., vol. 57, no. 6, pp. 1892-1905, 2010 .

[15] A. Accetta, M. Cirrincione, G. Marsala, M. Pucci, and G. Vitale, "PEM fuel cell system model predictive control and real-time operation on a power emulator," in Proc. of ECCE, 2010, pp. 1610-1616.

[16] T. Besselmann, J. Lofberg, and M. Morari, "Explicit MPC for LPV systems: Stability and optimality," IEEE Trans. Autom. Control, vol. 57, no. 9, pp. 2322-2332, 2012.

[17] B. M. Sanandaji, T. L. Vincent, A. Colclasure, and R. J. Kee, "Controloriented modeling of a solid-oxide fuel cell stack using an LPV model structure," in Proc. of the Dynamic Systems and Control Conference, 2009, pp. 793-800.

[18] D. Hernandez-Torres, "Commande robuste de générateurs électrochimiques hybrides," Ph.D. dissertation, Université de Grenoble, Grenoble, France, 2011.

[19] D. Hernandez-Torres, O. Sename, and D. Riu, "An LPV control approach for a fuel cell power generator air supply system," in P. Amer. Contr. Conf., 2012.

[20] S. D. Lira, V. Puig, J. Quevedo, and A. Husar, "LPV observer design for pem fuel cell system: Application to fault detection," J. Power Sources, vol. 196, no. 9, pp. 4298-4305, 2011.

[21] C. Kunusch, P. Puleston, M. Mayosky, and A. Husar, "Control oriented modelling and experimental validation of a PEMFC generation system," IEEE Trans. Energy Convers., vol. 6, no. 3, pp. 851-861, 2011.

[22] S. Lim and J. P. How, "Modeling and $H_{\infty}$ control for switched linear parameter-varying missile autopilot," IEEE Trans. Control Syst. Technol., vol. 11 , no. 6 , pp. 830-838, 2003.

[23] M. Chilali and P. Gahinet, " $H_{\infty}$ design with pole placement constraints: an LMI approach," IEEE Trans. Autom. Control, vol. 41, no. 3, pp. 358367, 1996.

[24] P. Apkarian and R. Adams, "Advanced gain-scheduling techniques for uncertain systems," IEEE Trans. Control Syst. Technol., vol. 6, no. 1, pp. 21-32, 1998.

[25] F. Wu, X. H. Yang, A. Packard, and G. S. Becker, "Induced L2-norm control for LPV systems with bounded parameter variation rates," Int. J. Robust Nonlinear Control, vol. 6, pp. 983-998, 1996.

[26] S. Tarbouriech and M. Turner, "Anti-windup design: an overview of some recent advances and open problems," IET Control Theory and A., vol. 3, no. 1, pp. 1-19, 2009.

[27] C. Roos, J.-M. Biannic, S. Tarbouriech, C. Prieur, and M. Jeanneau, "Onground aircraft control design using a parameter-varying anti-windup approach," Aerosp. Sci. Technol., vol. 14, no. 7, pp. 459-471, 2010.

[28] F. Forni and S. Galeani, "Gain-scheduled, model-based anti-windup for LPV systems," Automatica, vol. 46, no. 1, pp. 222-225, 2010.

[29] B. Lu, F. Wu, and S. Kim, "LPV antiwindup compensation for enhanced flight control performance," in Proc. of the AIAA Guidance, Navigation, and Control Conference, vol. 28, 2003, pp. 495-505.

[30] M. C. Turner and I. Postlethwaite, "A new perspective on static and low order anti-windup synthesis," Int.l J. Control, vol. 77, no. 1, pp. 27-44, 2004.

[31] W. Xie and T. Eisaka, "Design of LPV control systems based on Youla parameterisation," IEE P-Contr. Theor. Ap., vol. 151, no. 4, pp. 465-472, 2004.

[32] G. S. Becker and A. Packard, "Robust performance of linear parametrically varying systems using parametrically-dependent linear feedback," Syst. Control Lett., vol. 23, no. 3, pp. 205-215, 1994.

[33] P. Apkarian, P. Gahinet, and G. Becker, "Self-scheduled $H_{\infty}$ control of linear parameter-varying systems: a design example," Automatica, vol. 31, no. 9, pp. 1251-1261, 1995.

[34] R. Toth, P. S. Heuberger, and P. M. Van Den Hof, "Discretisation of linear parameter-varying state-space representations," IET Control Theory and A., vol. 4, no. 10, pp. 2082-2096, 2010.

[35] J. Sturm, "Using SeDuMi 1.02, a Matlab toolbox for optimization over symmetric cones," Optim. Method Softw., vol. 11-12, pp. 625-653, 1999.

[36] J. Löfberg, "Yalmip : A toolbox for modeling and optimization in MATLAB," in Proc. of the CACSD Conference, Taipei, Taiwan, 2004. 